\title{
Estresse ocupacional: estudo em um hospital filantrópico no estado de Minas Gerais
}

Maristela dos Santos Gomes

Mestre em Administração pelo Centro Universitário Unihorizontes

mariteladof@gmail.com

Luciano Zille Pereira

Doutor em Administração pela Universidade Federal de Minas Gerais. Professor Titular nos Cursos de Graduação. Mestrado Acadêmico em Administração do Centro Universitário Unihorizontes luciano.zille@unihorizontes.br

Pedro Favarini Aires de Lima

Mestrando em Administração pelo Centro Universitário Unihorizontes com bolsa vigente pela CAPES/CNPq.

pedro.lima@unihorizontes.br

\section{Editor Científico: José Edson Lara \\ Organização Comitê Científico \\ Double Blind Review pelo SEER/OJS \\ Recebido em 27.07.2018 \\ Aprovado em 15.11.2018}




\title{
Resumo
}

Este estudo objetivou descrever e explicar o estresse ocupacional na percepção de técnicosadministrativos que atuam em um hospital filantrópico no estado de Minas Gerais, sob a luz do Modelo Teórico de Explicação do Estresse Ocupacional, desenvolvido e validado por Zille (2005), adaptado para este estudo. Metodologicamente, caracterizou-se como estudo de caso descritivo e explicativo, de abordagem quantitativa. Os resultados apontaram que $70,3 \%$ dos técnicos-administrativos pesquisados apresentaram algum nível de estresse. Os sintomas prevalentes foram dor nos músculos do pescoço e ombros, ansiedade, dor de cabeça por tensão e fadiga. Para $17,1 \%$ e $48,6 \%$ dos pesquisados, respectivamente, observou-se tensões decorrentes do trabalho e das características pessoais. Por fim, observou-se que o aumento das fontes de tensão no trabalho e diminuição na utilização dos mecanismos de regulação ou estratégias de coping, leva ao aumento das manifestações do estresse ocupacional.

Palavras-Chaves: Estresse ocupacional; Técnicos-administrativos; Hospital; Hospital Filantrópico.

\section{a) Occupational stress: study at a philanthropic hospital in the state of Minas Gerais}

\begin{abstract}
This study aimed describe and explain occupational stress in the perception of technicians who work in a philanthropic hospital in the state of Minas Gerais under the theoretical model of explanation of occupational stress developed and validated by Zille (2005). The model was adapted for this study. Methodologically, it was characterized as a descriptive and explanatory case study, with a quantitative approach. The results showed that $70.3 \%$ of the administrative technicians surveyed presented some level of stress. The prevalent symptoms were pain in the muscles of the neck and shoulders, anxiety, tension headache and fatigue. For $17.1 \%$ and $48.6 \%$ of respondents, respectively, there were tensions due to work and personal characteristics. Finally, it was observed that the increase of the sources of tension in the work and decrease in the use of the regulation mechanisms or coping strategies, leads to the increase of the manifestations of the occupational stress.
\end{abstract}

Keywords: Occupational stress; Technical-administrative; Hospital; Philanthropic Hospital.

b) Estrés ocupacional: estudio en un hospital filantrópico en el estado de Minas Gerais

\section{Resumen}

Este estudio objetivó describir y explicar el estrés ocupacional en la percepción de técnicosadministrativos que actúan en un hospital filantrópico en el estado de Minas Gerais, bajo la luz del Modelo Teórico de Explicación del Estrés Ocupacional, desarrollado y validado por Zille (2005), adaptado para este estudio. Metodológicamente, se caracterizó como estudio de caso descriptivo y explicativo, de abordaje cuantitativo. Los resultados apuntaron que el 70,3\% de los técnicos-administrativos encuestados presentaron algún nivel de estrés. Los síntomas prevalentes fueron dolor en los músculos del cuello y los hombros, ansiedad, dolor de cabeza por tensión y fatiga. Para $17,1 \%$ y 48,6\% de los encuestados se observaron tensiones derivadas 
del trabajo y de las características personales. Por último, el aumento de las fuentes de tensión en el trabajo y disminución en la utilización de los mecanismos de regulación o estrategias de coping, lleva al aumento de las manifestaciones del estrés ocupacional.

Palabras clave: Estrés ocupacional; Técnicos-administrativos; Hospital; Hospital Filantrópico.

\section{Introdução}

O estresse é uma das manifestações que mais vem afetando a humanidade nos tempos atuais. Para os trabalhadores que atuam na área da saúde, além da necessidade de se incorporar aos avanços tecnológicos constantes, novas e maiores exigências vem desencadeando sentimentos de impotência e de ansiedade, colocando em risco a saúde física e emocional destes profissionais (Zille, 2005; Cavalheiro, 2008; Sousa \& Levi, 2010; Araújo, 2015; Moura; Greco; Arreguy-Sena; Martins \& Alves, 2016).

As prioridades das organizações, incluído as que atuam na área da saúde, têm sido modificadas e ajustadas para se adaptarem às novas demandas de uma sociedade cada vez mais exigente, dinâmica e complexa. Com evolução do pensamento administrativo e das ciências comportamentais, as organizações se despertam para o fato de que a atenção às pessoas é o grande diferencial, na medida em que os indivíduos constituem o principal ativo das instituições. É no indivíduo que está inserido o potencial de mudança, de aprimoramento e de inovação que as organizações precisam alcançar (Fischer, 2002).

No caso das instituições voltadas para a área de saúde, como os hospitais, também buscam com intensidade realizarem mudanças de natureza estrutural, tecnológica e cultural, para se adaptarem às demandas dos pacientes e do contexto econômico. Isso implica em modificações fundamentais no comportamento do trabalhador, nos padrões de trabalho e nos valores, afetando sua maneira de agir a partir do momento em que demanda novas atitudes e habilidades em relação ao trabalho (Newstrom \& Santos, 2008).

Nesse sentido, o uso de novas tecnologias intensificou o esforço gerado pelas organizações para elevar o nível da prestação de serviços, provocando a redefinição e novas formas de organização dos processos de trabalho, visando assim, melhores resultados, tanto para as organizações em si, como para a sociedade que demanda os serviços (Schermerhorn; Hunt \& Osborn, 2009; Oliveira \& Souza, 2012). 
Essas mudanças impactam a vida do trabalhador, que passou a vivenciar a angústia e o medo de ser excluído do mercado de trabalho enquanto procura se adaptar a estas exigências, que muitas vezes, causam pressão excessiva em suas atividades laborais, somado à complexidade de atuar em instituições da área de saúde, como os hospitais (Bendassolli \& Soboll, 2011; Gil-Monte, 2012).

Nessa direção, considerando a reestruturação organizacional, os modelos de gestão caracterizados pelo controle, centralização e hierarquização dão lugar a outros mais flexíveis (Braga \& Zille, 2010; Zille; Marques; Melo; Braga \& Zille, 2011).

Assim, à luz das mudanças, o gestor precisa atentar para o sentimento da equipe de trabalho, orientando e acompanhando o seu desenvolvimento, em direção às metas e objetivos traçados pela organização, voltados para a sua missão institucional (Newstrom \& Santos, 2008; Araújo, 2010; Melo; Cassini \& Lopes, 2011).

Considerando o setor da saúde, os impactos gerados em decorrência das transformações no contexto do trabalho, incidem sobre as práticas profissionais, uma vez que o advento das novas tecnologias requer novas habilidades para lidar com o grande aporte tecnológico, de equipamentos e processos. Pode ser considerado como fator relevante no desenvolvimento do trabalho dos técnico-administrativos que atuam em hospitais, podendo consistir em indutores de manifestações de estresse ocupacional (Jodas \& Haddad, 2009; Andolhe, 2009; Oliveira \& Souza, 2012; Santos, 2015).

A partir do contexto apresentado, este estudo objetivou descrever e explicar o estresse ocupacional na percepção de técnico-administrativos que atuam em um hospital filantrópico no estado de Minas Gerais.

\section{Referencial Teórico}

Selye $(1936,1959)$ observou que muitas pessoas sofriam de várias doenças físicas e a partir dai iniciou os primeiros estudos sobre as manifestações de estresse, publicando, em 1956, o livro The Stress of Life, no qual utilizou a palavra stress, para descrever as manifestações do corpo, relacionadas aos mecanismos bioquímico do estresse.

Os estudos realizados por Selye (1959) apontaram alguns sintomas como dores de cabeça, dores musculares, insônia, taquicardia, alergias, mudança de apetite, desânimo e fadiga. Essas reações, contudo, inicialmente, foram definidas como 'síndrome de se estar apenas 
doente'. Posteriormente, esses sintomas foram relacionados ao conceito que o autor denominou de stress, palavra esta que será grafada em português como 'estresse'.

Couto (2014) considera o estresse como manifestações derivadas de um desgaste anormal do organismo humano que resultam na diminuição da capacidade de trabalho do indivíduo diante das demandas psíquicas exigidas, consideradas acima de um limite suportável.

Limongi-França e Rodrigues (2005) apontam que o estresse deve ser observado não só como uma reação do organismo, mas também como uma relação particular entre uma pessoa, seu ambiente e as circunstâncias às quais está submetida. As fontes de tensão decorrentes, indutoras do estresse, devem ser avaliadas pelo indivíduo como uma ameaça ou exigências superiores à suas próprias habilidades ou recursos disponíveis, podendo colocar em perigo seu bem-estar físico e psíquico e, consequentemente, a sua sobrevivência.

Segundo Selye (1959) o conjunto de modificações não específicas que ocorrem no organismo, diante de situações de estresse, deu-se o nome de "Síndrome Geral de Adaptação" que, resumidamente, consiste em três fases: reação de alarme; resistência; e a última fase denominada de exaustão. Cada fase possui características específicas, sendo que na terceira, o indivíduo apresenta os diversos sintomas físicos e psíquicos relacionados ao estresse de forma muito intensa, inclusive podendo evoluir para quadros críticos de depressão e até para o óbito, dependendo do seu agravamento.

\subsection{Tipologias de estresse}

Para Limongi-França e Rodrigues (2005), as reações de estresse são naturais e até necessárias para a própria vida, no entanto, sob algumas circunstâncias, elas podem se tornar prejudiciais ao indivíduo. Dependendo da maneira como é observado e desenvolvido, o estresse se manifesta de diferentes formas como o eustresse e o distresse, estresse de sobrecarga e de monotonia, e o estresse agudo e crônico, que apresentam características específicas em suas manifestações.

Em relação ao eustresse ou estresse positivo, o esforço de adaptação gera sensação de realização pessoal, bem-estar e satisfação das necessidades, mesmo que decorrentes de esforços inesperados e até certo ponto desgastantes. É um esforço ‘sadio' na garantia de sobrevivência, ou seja, quando se faz algo agradável e os sentimentos são valorizados pelo esforço despendido, obtendo-se resultados positivos e recompensadores (Limongi-França \& Rodrigues, 2005; Couto, 2014). 
O distresse representa uma reação do organismo a um estímulo gerador de tensão com rompimento do equilíbrio biopsicossocial, ou seja, são situações disfuncionais do estresse que podem levar o individuo a diversas manifestações psíquicas e orgânicas, de uma forma geral, relacionadas ao sofrimento mental (Zille, 2005; Couto, 2014).

Outra forma de diferenciação refere-se ao estresse de sobrecarga e ao estresse de monotonia. No caso do estresse se sobrecarga, este ocorre quando a estrutura psíquica do indivíduo se torna incapaz de suportar as excessivas exigências psíquicas do meio por um período mais longo de tempo, ou seja, duas ou três semanas. Como decorrência, de uma forma geral, o individuo apresenta sintomas físicos e psíquicos, com reflexos negativos no seu desempenho profissional e nos demais ambientes da sua vida. No caso do estresse de monotonia, a situação é inversa, ou seja, o indivíduo é pouco estimulado em relação ao seu potencial de trabalho, muitas vezes subutilizado e submetido a situações incompatíveis e degradantes, considerando o seu potencial intelectual. Como decorrência do estresse de monotonia, os indivíduos tendem a desenvolver quadros de depressão, que inclusive, podem evoluir para o óbito (Zille \& Zille, 2010; Couto, 2014).

Em relação à sua duração, o estresse pode ser classificado como agudo ou crônico. $\mathrm{O}$ estresse agudo perdura por um período curto, horas, dias ou semanas e, depois, se dissipa. O estresse crônico, no entanto, apresenta sintomas com maior duração e intensidade, podendo causar maiores danos à saúde do indivíduo. Sujeita o indivíduo a um estado contínuo de tensão, muitas vezes crescente, não permitindo que o organismo se recupere a tempo para minimizar os desgastes decorrentes deste estado (Couto, 2014).

\subsection{O estresse no trabalho}

Quando no local de trabalho estão presentes as fontes causadoras do estresse, este é denominado estresse ocupacional ou estresse do trabalho. As manifestações associadas que são os sintomas, que podem ser físicos e/ou psíquicos, tendem a se desdobrarem em doenças decorrentes do estresse. Essas doenças, na sua maioria, são de origem psicossomática, podendo ocorrer manifestações como as mentais, as cardíacas, entre elas o infarto prematuro do miocárdio, gastrites, úlceras, dermatites, câncer, entre outras (Cooper et al., 1988; Karasek et al., 2000; Levi, 2005, 2010; Couto, 2014). 
Guerrer e Bianchi (2008) afirmam que o estresse no trabalho ocorre quando o ambiente laboral é visto como uma ameaça pelo trabalhador, repercutindo na sua vida profissional e tendo consequências em outros ambientes. Dessa forma, as demandas psíquicas existentes nesse ambiente tornam-se maiores do que a capacidade de tolerância psíquica que o indivíduo está preparado para enfrentar.

Dentre os efeitos negativos ou disfuncionais gerados nas organizações pelas consequências do estresse no trabalho, pode-se citar o aumento da rotatividade de pessoal, elevação do absenteísmo, maior incidência de acidentes no trabalho, aumento das licenças médicas, agravamento dos problemas decorrentes das relações humanas, como os conflitos, baixo comprometimento organizacional, desmotivação, e desejo de mudar de emprego com frequência, tendo como consequência final a queda da produtividade individual e dá organização (Lipp, 2001; Paschoal \& Tamayo, 2004; Zille, 2005; Zille et al., 2011).

\subsection{Fontes de tensão e sintomas de estresse}

As fontes de tensão no trabalho podem ser explicadas, segundo Zille (2005), por construtos relacionados aos processos de trabalho, relações no trabalho, nível de segurança proporcionado pelo ambiente laboral e a convivência com pessoas emocionalmente instáveis. Esses construtos, por meio de indicadores, podem refletir o nível de tensão decorrente do ambiente ocupacional que os trabalhadores são submetidos, medido por meio de indicadores como: realização de várias atividades ao mesmo tempo com alto grau de cobrança; trabalho intenso com cronogramas apertados; ambiente de trabalho tendo como filosofia a obsessão e compulsão por resultados e; a sobrecarga de trabalho ocasionada pelo uso intensivo de tecnologias como e-mails, softwares, sistemas integrados de gestão e produção, entre outros.

Outra fonte de tensão que também é importante e indutora do estresse ocupacional está relacionada às características individuais ou aspectos relacionados à personalidade dos indivíduos. As fontes de tensão relacionadas ao indivíduo referem-se às responsabilidades assumidas pelos trabalhadores acima dos limites técnicos e de aprendizagem recente, estilo e qualidade de vida, e os aspectos motivacionais relacionados ao trabalho. Essas fontes de tensão podem ser identificadas a partir dos seguintes indicadores: ritmo de vida; pensar e/ou realizar frequentemente duas ou mais atividades ao mesmo tempo, com dificuldade de concluí-las; não conseguir se desligar do trabalho, mesmo estando fora dele; ter que fazer atividades de trabalho bem acima da capacidade técnica e/ou atividades de aprendizado recente, das quais ainda não 
tem maturidade e domínio pleno; ter o dia muito tomado com uma série de compromissos assumidos, com pouco ou nenhum tempo livre; e por fim, ter os horários de descanso, após expediente, feriados e finais de semana, tomados pelo trabalho (Zille, 2005).

Em relação aos sintomas, estes são decorrentes do nível de tensão que os trabalhadores são submetidos na realização de suas atividades, ocorrendo ai um desequilíbrio psíquico, onde os indivíduos passam a manifestar sintomas, tanto de origem psíquica como física, que podem configurar como marcadores para a identificação dos quadros de estresse. Isso se dá em termos da frequência de cada sintoma, associado à sua intensidade. Os principais sintomas psíquicos são: ansiedade, nervosismo, angústia, irritabilidade, perda e/ou oscilação do senso de humor, ímpetos de raiva, depressão, síndrome do pânico, e mau humor (Cooper et al., 1988; Zille, 2005; Couto, 2014).

Em relação aos sintomas físicos, que podem ocorrer concomitantemente aos sintomas psíquicos, os mais evidentes são: fadiga, dor nos músculos do pescoço e ombros, dor de cabeça por tensão, falta ou excesso de apetite, indisposição gástrica, insônia, palpitações, dor discreta no peito sobtensão, tontura/vertigem, desmaio, enxaqueca, dificuldade de concentração e memorização, baixa imunidade, diminuição do desejo sexual, entre outros. Os sintomas, tanto os físicos como os psíquicos, dependendo da frequência e intensidade podem desencadear doenças, sendo as mais comuns relacionas ao estresse as cardíacas, com ênfase para o infarto prematuro do miocárdio, doenças mentais, úlcera, gastrite, doenças alérgicas, fibromialgia, eczema, psoríase, urticária, alopecia, câncer, entre outras (Couto, 1987, 2014; Cooper et al., 1988; Zille, 2005; Levi, 2003, 2005, 2008, 2010).

Zille $(2005,2011)$ aponta que a relação de agentes ambientais, socioeconômicos e culturais provenientes do modelo econômico industrial e capitalista presente na sociedade, vêm contribuindo para aumentar as situações tensionantes no ambiente de trabalho, atuando de forma a precipitar sintomas e consequentes doenças relacionadas ao estresse ocupacional.

\subsection{Mecanismos de regulação (Coping)}

De acordo com Limongi-França e Rodrigues (2005), os mecanismos de regulação são um conjunto de esforços que uma pessoa desenvolve para manejar ou lidar com as demandas externas ou internas, que são avaliadas por ela como excessivas ou acima de suas possibilidades. 
Para Savoia (1999), as habilidades desenvolvidas para o domínio das situações de estresse e adaptação também são denominadas coping. O coping é definido como todos os esforços de controle, ou seja, é uma resposta ao estresse, comportamental e/ou cognitiva, com a finalidade de reduzir ou eliminar os sintomas aversivos provocados pelos estressores. Tratase de uma ação transacional entre a pessoa e o ambiente, onde as características de personalidade de cada um desempenha papel importante no processo de enfrentamento ao estresse.

Para Zille $(2005,2011)$ e Couto (2014), o estresse ainda pode ser combatido de três formas: controlar ou eliminar as fontes de tensão nos seus diversos ambientes; possibilitar ao indivíduo maior robustez em termos da sua estrutura de personalidade para estar mais apto a absorver os estressores; e, num terceiro plano, utilizar estas duas estratégias de forma concomitante.

\subsection{Modelo teórico de explicação do estresse ocupacional (MTEG)}

O modelo teórico utilizado para explicar o estresse ocupacional, foi desenvolvido e validado por Zille (2005) e adaptado para analisar o estresse ocupacional neste estudo, onde os sujeitos foram os técnico-administrativos que atuam em um hospital filantrópico. Este modelo, já adaptado, também foi utilizado por Santos (2015) e Gonçalves (2016) para estudarem servidores que atuavam em um hospital público universitário no estado de Minas Gerais.

Em relação ao modelo em referência, foi estruturado em quatro construtos: fontes de tensão no trabalho, fontes de tensão do indivíduo, sintomas de estresse e mecanismos de regulação ou estratégia de coping. As adaptações se deram em relação aos termos utilizados na área de saúde e em relação ao construto 'fontes de tensão do indivíduo' onde se retirou a variável 'gerente'.

De acordo com os indicadores do modelo, a ausência de estresse indica bom equilíbrio entre a estrutura psíquica do indivíduo e as pressões que ele sofre em seu ambiente laboral. $\mathrm{O}$ estresse leve/moderado indica a ocorrência de manifestações de estresse, no entanto em grau compensado, podendo não gerar impactos importantes para os indivíduos nos planos físico e psicológico. Já o estresse intenso, por sua vez, o indivíduo já convive com os principais sintomas de estresse de forma importante, apresentando problemas de concentração e/ou dificuldade para realização de atividades que antes eram executadas com facilidade. Por fim, o estresse muito intenso é indicador de estado bastante grave, onde o indivíduo perde quase que $@$ Revista Gestão \& Tecnologia, Pedro Leopoldo, v. 18, n. 3, p. 204-225,set./dez. 2018 
totalmente a sua capacidade de trabalho. É eminente a necessidade de tratamento clinico e psicológico, visando à moderação ou eliminação do desequilíbrio da relação entre a estrutura psíquica e as pressões psíquicas advindas do ambiente ocupacional (Zille, 2005).

Ainda de acordo com Zille (2005), os construtos, fontes de tensão no trabalho, fontes de tensão do indivíduo, levam ao construto sintomas, que por sua vez é mediado pelo construto mecanismos de regulação (coping)

A partir dessa relação, este estudo buscou avaliar a seguinte hipótese: H1: maiores níveis de fontes de tensão no trabalho e de fontes de tensão do indivíduo e menores níveis de atuação dos mecanismos de regulação levam a maiores níveis de estresse ocupacional.

\section{Metodologia}

A pesquisa realizada caracterizou-se como descritiva e explicativa, de abordagem quantitativa por meio de estudo de caso quantitativo.

Para Cervo e Bervian (2002), a pesquisa descritiva visa descrever as características de determinada população ou fenômeno, bem como o estabelecimento de relações entre as variáveis estudadas e para Gil (2010), a pesquisa explicativa identifica os fatores que determinam ou que contribuem para a ocorrência dos fenômenos, aprofundando o conhecimento da realidade, uma vez que explica a razão e o porquê dos fenômenos. Dessa forma, a pesquisa buscou descrever e explicar o estresse ocupacional na percepção dos técnicoadministrativos que atuam em um hospital filantrópico localizado no estado de Minas Gerais.

Em relação à abordagem esta foi de natureza quantitativa, que segundo Malhotra (2001) procura quantificar os dados, aplicando alguma forma da análise estatística. Em relação ao método, utilizou-se o estudo de caso quantitativo, de acordo com Yin (2005), é uma estratégia de pesquisa flexível, que permite ao pesquisador utilizar diferentes técnicas de levantamento de dados, visando ampliar a interpretação das informações obtidas.

A população foi constituída por 138 técnico-administrativos e a amostra calculada com base em Barnett (1982) e erro amostral de 5\%, alcançou o índice necessário de 97 indivíduos. A amostra, por sua vez, contou com 111 questionários válidos, o que possibilitou a realização de todas as análises estatísticas previstas com segurança.

A coleta de dados foi realizada por meio da aplicação de questionário aderente ao modelo teórico de análise utilizado, desenvolvido e validado por Zille (2005), adaptado para esta 
pesquisa. $\mathrm{O}$ questionário em referência foi estruturado em cinco partes conforme descrito a seguir: dados demográficos, funcionais, hábitos de vida e saúde; sintomas de estresse; fontes de tensão no trabalho e do indivíduo; indicadores de impacto no trabalho e mecanismos de regulação.

O processamento dos dados foi realizado com base Software estatístico IBM SPSS versão 20.0.0. A análise iniciou-se com a avaliação de dados ausentes, extremos, média, mediana, desvio padrão, mínimo e máximo de cada variável. Por fim, para avaliar a relação entre o estresse ocupacional e as fontes de tensão no trabalho, fontes de tensão do indivíduo e mecanismos de regulação, foram realizadas análises de correlação não paramétrica de Spearman e regressão linear múltipla.

Antes de realizar a análise de regressão múltipla, avaliou-se a correlação entre as variáveis do estudo, uma vez que ambas são baseadas em uma relação linear (Hair; Black; Babin; Anderson \& Tatham 2005).

Teve-se como parâmetros o coeficiente de correlação que varia de um negativo até um positivo. De acordo com Cohen (1988), os valores entre 0,10 e 0,29 podem ser considerados pequenos, escores entre 0,30 a 0,49 podem ser tidos como médio e valores entre 0,50 e 1 são considerados como grandes.

Conforme Hair et al., (2005), a análise de regressão objetivou prever mudanças na variável dependente de acordo com alterações em mais de uma variável independente (regressão múltipla). Realizou-se análise em relação ao tamanho da amostra e observou-se o método computacional empregado. Conforme Field (2009), o valor da amostra foi calculado pela fórmula $50+8 \mathrm{k}$, onde se estabeleceu o número de previsores. No caso desta pesquisa, o número mínimo seria de 74 indivíduos, sendo que a amostra constou de 111 questionários válidos. Teve-se, portanto, segurança para realização da regressão em relação a este ponto. Quanto ao método, optou-se pelo 'enter' e a sua escolha ocorreu diante do fato de que este método emprega uma perspectiva confirmatória, uma vez que os pesquisadores especificaram o conjunto de variáveis independentes a serem consideradas no modelo (Hair et al., 2005).

\section{Apresentação e Discussão dos Resultados}

Com relação à descrição do perfil da amostra no que se refere ao gênero, observou-se que $85,6 \%$ são do sexo feminino e 14,4\% são do sexo masculino e em relação à faixa etária, $45,0 \%$ têm entre 26 e 35 anos. 
No que se refere ao estado civil, 50,0\% são casados ou vivem com cônjuge e 40,9\% solteiros e 5,5\% declararam-se viúvos. Para o cargo ocupado, os maiores porcentagens foram observados em relação a coordenador técnico $(12,1 \%)$ e auxiliar de farmácia $(10,3 \%)$. Considerando o setor de trabalho, as maiores incidências recaíram na farmácia $(16,5 \%)$ e na unidade de alimentação $(14,6 \%)$.

Quanto ao tempo de trabalho, a maioria, 80,2\%, realizam atividades no hospital entre 1 a 5 anos e os demais, acima de 5 anos. No que se refere à jornada de trabalho, 52,3\% afirmaram trabalhar 40 horas semanais e $2,8 \%, 30$ horas.

Quanto à escolaridade, a maioria, 64,2\%, possui ensino médio completo; 24,5\%, graduação completa; $10,4 \%$, graduação incompleta; e $0,9 \%$, ensino fundamental. No que se refere à pós-graduação, $7,2 \%$ e 6,3\% dos respondentes afirmam possuir, respectivamente, especialização incompleta e completa. Tem-se, ainda, que 1,8\% dos pesquisados possuem mestrado e $0,9 \%$ doutorado.

Em relação ao hábito de vida, tem-se que $93,7 \%$ dos respondentes declaram não fumar. Quando questionados a respeito do consumo de bebida alcoólica, mais da metade, ou seja, $54,5 \%$ responderam positivamente. No que se refere à relação entre o consumo de bebida alcoólica e o estresse ocupacional, foi possível perceber que os técnicos que fazem uso de bebida alcoólica, foram aqueles que apresentaram a maior proporção entre os indivíduos com quadros de estresse.

No que se refere a problema de saúde, 27,5\% afirmam possuir alguma enfermidade. As que apresentaram as maiores incidências foram hipertensão $(27,3 \%)$ e gastrite $(21,2 \%)$. Em relação às doenças identificadas, a hipertensão e a gastrite têm sido apontadas em estudos por parte de alguns pesquisadores como miniestações que podem estar relacionadas aos quadros de estresse (Cooper et al.,1988; Karasek et al., 2000; Levi, 2005, 2010; Couto, 2014).

\subsection{Análise do estresse ocupacional}

Para análise do estresse ocupacional, calculou-se uma média ponderada dos sintomas e os indivíduos foram agrupados em níveis de estresse conforme proposição de Zille (2005): médias de 1,00 a 1,74 indicaram ausência de estresse; 1,75 a 2,45, estresse leve/moderado; 2,46 a 3,15, estresse intenso; e 3,16 a 5,00, estresse muito intenso, numa escala variando de 1,0 a 5,0 . Os resultados globais obtidos em relação ao estresse no trabalho dos técnicos pesquisados 
são apresentados por meio da Tabela 1. Observa-se que 70,3\% dos respondentes foram identificados com manifestação de estresse, que varia de leve/moderado a estresse muito intenso.

Desses, os casos mais críticos recaem em 7,2\% dos técnicos que apresentam manifestações de estresse nos níveis intenso e muito intenso. Para esses indivíduos é indicando a necessidade de tratamento clínico e psicológico, com vista a controlar o desequilíbrio entre estrutura a psíquica e as pressões oriundas do ambiente de trabalho, além da instituição analisar o contexto ocupacional que estes trabalhadores estão submetidos. Para os demais, 63,1\%, o estresse manifesta em nível leve/moderado, onde a ocorrência de estresse se estabelece de modo compensado, no entanto, se agravado, poderá ocasionar impactos de ordem psíquica e orgânica, com comprometimento da saúde e reflexos na capacidade produtiva desses trabalhadores.

\section{Tabela 1}

Análise do nível de estresse

\begin{tabular}{|c|c|c|c|c|c|c|c|c|}
\hline \multirow[b]{2}{*}{ Nível de Estresse } & \multicolumn{3}{|c|}{ Frequência } & \multirow[b]{2}{*}{ Média } & \multirow[b]{2}{*}{ Mediana } & \multirow{2}{*}{$\begin{array}{l}\text { Desvio } \\
\text { padrão }\end{array}$} & \multirow[b]{2}{*}{ Min. } & \multirow[b]{2}{*}{ Max. } \\
\hline & $\mathbf{N}$ & $\%$ & \% acumulado de estresse & & & & & \\
\hline Ausência de estresse & 33 & 29,7 & -- & 1,40 & 1,39 & 0,20 & 1,00 & 1,68 \\
\hline Leve a moderado & 70 & 63,1 & 63,1 & 1,96 & 1,87 & 0,17 & 1,76 & 2,43 \\
\hline Estresse intenso & 6 & 5,4 & 68,5 & 2,79 & 2,78 & 0,19 & 2,50 & 3,05 \\
\hline Estresse muito intenso & 2 & 1,8 & 70,3 & 3,64 & 3,64 & 0,12 & 3,55 & 3,72 \\
\hline Análise global & 111 & 100,0 & 70,3 & 1,87 & 1,87 & 0,45 & 1,00 & 3,72 \\
\hline
\end{tabular}

Fonte: Dados da pesquisa, 2017.

Resultados semelhantes foram encontrados por Santos e Zille (2014) em pesquisa com técnicos de enfermagem em um hospital público federal de Minas Gerais, bem como por Gonçalves (2016), que também pesquisou trabalhadores que atuam em hospital público. Os primeiros autores identificaram que $71 \%$ dos profissionais apresentavam quadro de estresse ocupacional e o segundo $74 \%$. Como podem ser observados, os resultados do presente estudo se aproxima muito de resultados de pesquisas recentes, realizadas em um mesmo lócus de trabalho, ou seja, o hospital, utilizando o mesmo modelo teórico para análise.

Em síntese, observa-se que a maioria dos pesquisados apresentam quadros de estresse que devem ser monitorados e gerenciados pela instituição. Destaca-se o contingente de 7,2\%, que mantêm níveis de estresse intenso ou muito intenso, consistindo em casos mais críticos, cuja intervenção deverá ser de imediato. A continuidade desses quadros afetará tanto os 
indivíduos quanto a instituição em relação ao resultado do trabalho, que consiste em atender a comunidade no que se refere aos episódios relacionados à saúde.

\subsubsection{Sintomas de estresse}

Para análise dos sintomas de estresse, os pesquisados foram novamente agrupados. Aqueles classificados com ausência de estresse foram mantidos neste grupo e os categorizados com alguma manifestação de estresse foram reagrupados na categoria algum nível de estresse. Posteriormente, avaliaram-se os indivíduos que obtiveram marcações frequente e muito frequente em cada um dos indicadores avaliados. Observou-se que mais de um quinto dos indivíduos classificados com algum nível de estresse manifestam dor nos músculos do pescoço e ombros, ansiedade, dor de cabeça por tensão e fadiga. Dentre os indivíduos com ausência de estresse, poucos sintomas foram manifestados em relação àqueles com estresse.

Para Levi (2005, 2010), Karasek et al., (2000) e Couto (2014), o estresse pode desencadear outros episódios, entre eles doenças relacionadas ao sistema cardiovascular, hipertensão arterial sistêmica, doenças gástricas entre outras.

Resultados similares foram encontrados por Santos e Zille (2014) em pesquisa realizada com técnicos de enfermagem em um hospital público. Nesse estudo, os principais sintomas apresentados foram dor nos músculos do pescoço e ombros, ansiedade, fadiga e nervosismo.

Em síntese, observou-se que todos os sintomas relacionados ao estresse ocupacional foram significativamente mais frequentes e intensos no grupo de técnicos com algum nível de estresse, se comparado ao grupo de técnicos que não apresentaram manifestações de estresse.

\subsection{Fontes de tensão}

As fontes de tensão no trabalho e do indivíduo estão relacionadas aos construtos do MTEG. Fontes de tensão no trabalho: processos de trabalho, relações no trabalho, segurança nas relações de trabalho e convivência com indivíduos de personalidade difícil. Fontes de tensão do indivíduo: responsabilidades acima dos limites, estilo e qualidade de vida, e desmotivação.

Para análise das fontes de tensão, calculou-se a média dos indicadores conforme proposto por Zille (2005). As referidas médias foram analisadas e os indivíduos foram 
agrupados em três níveis diferentes de tensão: ausência de tensão (médias entre 1,00 e 2,18), tensão moderada (médias entre 2,19 e 3,80) e tensão intensa (médias entre 3,81 a 5,00).

Em relação às fontes de tensão no trabalho, tem-se que 15,3\% e 1,8\% mantêm, respectivamente, níveis de tensão moderada e intensa. Dentre os pesquisados e classificados com algum nível de estresse, as maiores incidências foram observadas quanto à cobrança para se produzir cada vez mais com o mínimo de recursos e conviver com indivíduos com algum nível de desequilíbrio emocional.

Para aqueles que não apresentam estresse, essas porcentagens diminuiram, respectivamente, para $6,5 \%$ e $3,1 \%$. As ocorrências identificadas para este grupo referem-se a relacionamentos interpessoais de má qualidade no ambiente de trabalho e cobranças excessivas pela utilização de menos recursos materiais para entrega de cada vez mais resultados de trabalho.

Dessa forma, concluiu-se que as fontes de tensão que foram evidenciadas, devem passar por criteriosa análise por parte da instituição pesquisada, visando minimizar ou eliminar as situações tensionantes de origem do trabalho, como indutoras dos quadros de estresse identificados neste estudo.

Os pesquisados foram indagados, também, a respeito de quais seriam as situações mais importantes para que o ambiente de trabalho se tornasse menos tenso e estressante. As estratégias mais citadas foram melhorias na comunicação e nos relacionamentos interpessoais.

Em relação às fontes de tensão do indivíduo, observou-se que 43,2\% encontram-se sobtensão moderada nesta categoria de análise. A questão mais crítica recai sobre $5,4 \%$ da amostra que manifestou tensão intensa em relação a essa variável. Dentre o grupo com manifestação de estresse, verificou-se que os indicadores com as maiores porcentagens remetem ao fato de se levar a vida de forma muito corrida, ter o dia tomado por compromissos com pouco tempo livre e desempenhar várias atividades concomitantes, com supressão do tempo livre para descanso. Dessa forma, a diminuição do nível de estresse dos pesquisados passa pela "desaceleração" do ritmo de vida e pela dispensa de tempo para descanso e lazer.

Sabe-se que o ambiente hospitalar é instável, propício a reclamar a presença de seus funcionários a qualquer momento. O trabalhador, mesmo em seu momento de folga, fica atento a chamadas que podem convocá-lo para retorno ao trabalho por situações diversas. Esse profissional vive em estado de alerta e tensão e, de alguma forma, leva para casa as sensações vividas no ambiente de trabalho, o que também contribui para aumentar o nível de estresse. Os plantões mudam a rotina de sono, surgindo, na maioria das vezes, desequilíbrios físicos e $@$ Revista Gestão \& Tecnologia, Pedro Leopoldo, v. 18, n. 3, p. 204-225,set./dez. 2018 
emocionais capazes de transformar os trabalhadores em pessoas além de estressadas, doentes (Andolhe, 2009; Sousa \& Araújo, 2015; Azevedo; Nery \& Cardoso; 2017).

\subsection{Mecanismos de regulação (coping)}

Os mecanismos de regulação ou estratégias de enfrentamento ao estresse são práticas adotadas pelos técnicos que, de certa forma, amenizam as situações tensionantes vivenciadas na realização do trabalho. Para avaliação dessa variável, calculou-se um indicador ancorado na média deste construto e os indivíduos foram agrupados em três categorias de relevância: relevante (médias entre 1,00 e 2,43), moderadamente relevante (médias entre 2,44 e 3,55) e irrelevante (médias entre 3,56 e 5,00).

Verificou-se que, para a maioria dos pesquisados, a atuação dos mecanismos de regulação ocorre em nível moderadamente relevante $(57,7 \%)$. Um percentual significativo de indivíduos acredita que os mecanismos de regulação são relevantes $(39,6 \%)$ e apenas 2,7\% acreditam que a atuação dos mecanismos se mostra irrelevante. Esse resultado sugere que os gestores deveriam considerar esses mecanismos como formas de catarse, ou seja, de liberação das sensações que produzem danos ao trabalhador, transformando o ambiente laboral em ambiente de prazer.

Observou-se que no grupo de indivíduos que não apresentou manifestações de estresse, todos os indicadores foram identificados como presentes e utilizados por pelo menos metade dos pesquisados. Essa constatação sugere que a maior atuação dos mecanismos de regulação vem acompanhada de diminuição dos níveis de estresse, o que, consequentemente, tem relação com o bem-estar dos profissionais, impactando positivamente na saúde destes trabalhadores e, consequentemente, no desempenho do trabalho.

\subsection{Relação do estresse com as fontes de tensão no trabalho, do indivíduo e mecanismos de regulação}

Para verificação da relação entre estresse ocupacional e fontes de tensão no trabalho, fontes de tensão do indivíduo e mecanismos de regulação, realizou-se análises de correlação e de regressão linear. Os resultados indicaram existência de correlações de média intensidade entre estresse ocupacional e fontes de tensão no trabalho (ro $=0,414$ ), fontes de tensão do 
indivíduo (ro = 0,366) e mecanismos de regulação (ro = -0,312). Todas as correlações indicadas são significativas ao nível de $1 \%$ e o p encontrado em todos os casos foi $<0,001$.

Prosseguiu-se a realização da análise de regressão para testar a hipótese aventada nesta pesquisa. Nesse caso, considerou-se o estresse como variável dependente e as fontes de tensão no trabalho, fontes de tensão do indivíduo e mecanismos de regulação, como independentes. As relações citadas alicerçam-se nos estudos de Zille (2005).

Os resultados da análise de regressão indicaram que mecanismos de regulação e fontes de tensão no trabalho impactam os níveis de estresse ocupacional $(\mathrm{F}(3,107)=14,606$; $\mathrm{p}<$ 0,000), sendo a primeira delas a mais relevante. A variável fonte de tensão do indivíduo não ingressou no modelo. Supõe-se que essa exclusão se ancora na correlação existente entre ela e fontes de tensão no trabalho $(r=0,491, \mathrm{p}<0,001)$, o que faz da inclusão das fontes individuais, esforço redundante na previsão.

O sumário do modelo de regressão encontra-se na Tabela 2. Pode-se inferir que 27,1\% das variações no estresse ocupacional são explicadas pelas variações nas fontes de tensão do trabalho e nos mecanismos de regulação. O percentual encontrado revela-se adequado, uma vez que, nas ciências sociais aplicadas, o coeficiente de determinação ajustado gira em torno de 30\% (Marques; Borges \& Reis, 2015).

\section{Tabela 2}

Sumário do modelo de regressão entre estresse ocupacional e fontes de tensão no trabalho, do indivíduo e mecanismos de regulação

\begin{tabular}{llllll}
\hline \hline Modelo & $\mathbf{R}$ & R quadrado & R quadrado ajustado & Erro padrão da estimativa & Durbin-Watson \\
\hline 1 & $0,539 \mathrm{a}$ & 0,291 & 0,271 & 0,3897 & 1,569 \\
\hline \hline
\end{tabular}

a. Preditores: (Constante), mecanismos de regulação, fontes de tensão do indivíduo e fontes de tensão no trabalho

b. Variável Dependente: Estresse ocupacional

Fonte: Dados da pesquisa, 2017.

Portanto, incrementos nas fontes de tensão no trabalho e diminuição na utilização dos mecanismos de regulação levam a aumentos no estresse ocupacional. Assim, a Hipótese 1 deste estudo é parcialmente confirmada, uma vez que o modelo proposto não contemplou a variável fontes de tensão do indivíduo.

A validade dos resultados encontrados na regressão linear múltipla, segundo Pestana e Gagueiro (2000), depende da verificação de cinco pressupostos. Cada um deles foi atestado para esta pesquisa conforme explicações a seguir: (a) linearidade, dada à existência de 
correlações significativas entre a variável dependente e as independentes; (b) normalidade dos resíduos, uma vez que o teste Kolmogorov Smirnov resultou em p > 0,200; (c) inexistência de auto correlação dos resíduos, dado que o valor de Durbin Watson $(1,569)$ encontra-se próximo de 2; (d) homocedasticidade dos resíduos, considerando que os pontos do gráfico de dispersão entre resíduos estudantizados e os valores preditos padronizados mantêm uma amplitude aproximadamente constante em relação ao eixo horizontal; (e) multicolinearidade, uma vez que os valores de tolerância $(0,697,0,758$ e 0,885$)$ e de VIF $(1,435,1,130$ e 1,319) encontram-se distantes dos limites propostos por Hair et al., (2005) (tolerância abaixo 0,10 e VIF acima de 10). Assim, todos os pressupostos pertinentes à regressão foram confirmados e seus resultados válidos para a população deste estudo.

\section{Conclusões}

Esse estudo cumpriu o objetivo de descrever e explicar o estresse ocupacional na percepção de técnico-administrativos que atuam em um hospital filantrópico, no estado de Minas Gerais.

Em termos teóricos o estudo ancorou-se no MTEG, permitindo identificar os sintomas e o nível de estresse dos profissionais pesquisados, classificando-os por intensidade; as fontes de tensão que levaram ao desenvolvimento de quadros de estresse e; os mecanismos de regulação utilizados para minimizar ou eliminar tais manifestações.

Em termos metodológicos a pesquisa foi descritiva e explicativa de abordagem quantitativa, utilizando-se como estratégia o método de estudo de caso quantitativo. A população foi constituída por 138 sujeitos com amostra de 111 indivíduos.

Os dados foram analisados com base na estatística descritiva (frequência relativa, absoluta e acumulada; média; mediana; desvio padrão; mínimo e máximo) e inferencial, por meio de análise de regressão linear múltipla.

Em termos de resultados, no que se refere ao perfil demográfico e funcional, observouse que a maioria da amostra foi composta por mulheres, por indivíduos com idade entre 26 e 35 anos, casados ou que vivem com cônjuge, e que trabalham na instituição entre um e cinco anos, cumprindo jornada de 40 horas semanais e possui ensino médio completo. 
Grande parte dos pesquisados não fumam e um pouco mais da metade consome bebida alcoólica. Aproximadamente um terço, apresentam enfermidades tais como hipertensão e gastrite.

Em relação às manifestações do estresse ocupacional, tem-se que 70,3\% dos pesquisados apresentam esse quadro em intensidades que variaram de leve/moderada a muito intensa. Para redução desses níveis, dentre outros, os pesquisados apontaram ser necessário melhorar a comunicação entre as pessoas e os departamentos, de modo que os objetivos do trabalho sejam mais transparentes para todos. Apontaram também, a necessidade de melhoria no relacionamento interpessoal, buscando maior compreensão e respeito entre os funcionários e a promoção do espírito de equipe.

Quanto às fontes de tensão no trabalho, observou-se que aproximadamente um quinto dos pesquisados encontram-se sobtensão. Os fatores que mais se evidenciaram foram à cobrança para se produzir mais com recursos mínimos; e conviver com indivíduos que apresentam comprometimento emocional disfuncional. Indicaram, ainda, que os fatores mais difíceis de serem mudados na Instituição e que culminam em elevar a tensão no ambiente de trabalho são a pouca disponibilidade de recursos financeiros por parte da instituição e a falta de incentivo salarial, aliado à perspectiva de carreira funcional.

Observou-se, ainda, que quase a metade dos respondentes apresentou tensão relacionada às características individuais, em intensidade que variou de moderada a intensa. Os principais indicadores foram levar a vida de forma muito corrida, realizando cada vez mais trabalho em menos tempo e ter o dia muito tomado por compromissos, com pouco ou nenhum tempo livre.

No que se refere às estratégias pessoais para se reduzir o impacto de situações tensionantes no ambiente de trabalho foi apontada pelos pesquisados a prática de controle emocional, ouvir música e apoio social de familiares e amigos.

Dados da pesquisa revelaram ainda haver uma relação significante entre estresse e fontes de tensão no trabalho e mecanismos de regulação. Incrementos nas fontes de tensão no trabalho e diminuição na utilização dos mecanismos de regulação levam a aumentar a incidência de manifestações de estresse e a sua intensidade, confirmando parcialmente a hipótese aventada no estudo.

Em termos acadêmicos, as contribuições giram em torno da confirmação do Modelo Teórico Explicativo do Estresse Ocupacional (Zille, 2005) como modelo adequado para analisar o estresse ocupacional. 
As contribuições do estudo relacionam ainda a discussão que se estabelece entre estresse ocupacional e seus preditores em profissionais técnico-administrativos de um hospital. Os resultados aqui encontrados poderão servir de subsídios para criação de programas a fim de mitigar os níveis de estresse e de tensão encontrados entre os profissionais e de fomentar a utilização de mecanismos de regulação. Contribui também para o avanço do conhecimento multidisciplinar em relação à saúde do trabalhador, fornecendo subsídios visando promover estudos para aplicação de medidas preventivas para redução do estresse no trabalho. Podem ainda contribuir para aperfeiçoamento, quanto às adequações estruturais, organizacionais e revisão das políticas de gestão de pessoas.

Por fim, as limitações desta pesquisa giram em torno do fato de que ela trata apenas da análise dos profissionais técnico-administrativos da Instituição pesquisada. Nesse sentido, pesquisas futuras podem investigar os demais profissionais que atuam no Hospital pesquisado. Pesquisas posteriores podem também, avaliar se as fontes de tensão do indivíduo, de fato, não impactam o estresse ocupacional ou se essa condição se restringe apenas à população aqui considerada. Assim, estudos futuros podem expandir e aprofundar o conhecimento sobre o tema estudado, por se trata de um grande dilema para trabalhadores e organizações em todo o mundo.

\section{Referências}

Andolhe, R. (2009). Stress e coping da equipe de enfermagem no cuidado à mulher com câncer de mama. Dissertação de Mestrado não-publicada, Universidade Federal de Santa Maria, Santa Maria, Brasil.

Araújo, L. C. G. (2010). Organização, sistemas e métodos e novas tecnologias de gestão organizacional. São Paulo: Atlas.

Azevedo, B. D. S., Nery, A. A., Cardoso., J. P. (2017). Occupational stress and dissatisfaction with quality of work life in nursing. Texto \& Contexto-Enfermagem, 26(1).

Barnett, V. (1982). Sample survey: principles and methods. London: Arnold.

Bendassolli, P. F., Soboll, L. A. P. (2011). Clínicas do trabalho: novas perspectivas para compreensão do trabalho na atualidade. São Paulo: Atlas.

Braga, D. C., Zille, L. P. (2010). Estresse ocupacional e as principais fontes de tensão no trabalho: impactos na produtividade de gestores do setor de energia elétrica brasileiro. In Encontro da Associação Nacional de Pós-Graduação e Pesquisa em Administração - EnANPAD, Rio de Janeiro.

Bunker, S. J., et al. (2003). "Stress" and coronary heart disease: psychosocial risk factors. The Medical Journal of Australia, 178(6), 272-276.

Cavalheiro, A. M. (2008). Estresse em enfermeiros com atuação em unidades de terapia intensiva. Tese de Doutorado não-publicada, Escola de Enfermagem da Universidade de São Paulo, São Paulo, Brasil.

Cervo, A. L., Bervian, P. A. (2002). Metodologia científica. São Paulo: Prentice Hall. 
Chamon, E. M. Q. O., Marinho, R. C., Oliveira, A. L. (2006). Estresse ocupacional, estratégias de enfretamento e Síndrome de Burnout: um estudo com a equipe de enfermagem de um hospital privado do estado de São Paulo. In Enanpad, Salvador.

Cohen, A. (2003). Multiple commitment in the workplace: an integrative approach. Mahwah, NJ: Lawrence Erlbaum.

Cooper, C. L. et al. (1988). Occupational stress indicator: test sources of pressure in job. Austin: Windsor.

Couto, H. A. (1987). Stress e qualidade de vida dos executivos. Rio de Janeiro: Cop.

Couto, H. A. (2014). Ergonomia do corpo e cérebro no trabalho: os princípios e a aplicação prática. Belo Horizonte: Ergo.

Dolan, S. (2006). Estresse, autoestima, saúde e trabalho. Rio de Janeiro: Qualitymark.

Field, A. Descobrindo a estatística usando o SPSS. (2th ed.). Porto Alegre: Artmed.

Fischer, A. L. (2002). Um resgate conceitual e histórico dos modelos de gestão de pessoas. In França, A. C. L., Fleury, M. T. L. (Ed.). As pessoas na organização. São Paulo: Editora Gente.

Gil-Monte, P. R. (2012). Riesgos psicosociales en el trabajo y salud ocupacional. Peru Med Exp Salud Publica. 29(2), 237-241.

Gil, A. C. (2010). Como elaborar projetos de pesquisa. São Paulo: Atlas.

Goldberg, P. (1986). A saúde dos executivos: como identificar sinais de perigo para a saúde e levar a melhor contra o estresse. Rio de Janeiro: Guanabara.

Gonçalves, A. S. (2016). ESTRESSE OCUPACIONAL: estudo com gestores de um hospital público universitário do estado de Minas Gerais. Dissertação de Mestrado não-publicada, Centro Universitário Unihorizontes, Belo Horizonte, Brasil.

Moura, D. C. A., Greco, R. M., Arreguy-Sena, C., Martins, N. A., Alves, M. S. (2016). Labor conditions and theory of Betty Neuman: third-party workers of a public university. Journal of Nursing UFPE on-line, 10(2), 727-735.

Guerrer, F. J. L., Bianchi, E. R. F. (2008). Caracterização do estresse nos enfermeiros de unidade de terapia intensiva. Revista de Enfermagem da USP, 42(2). 355-362.

Hair, J. F., Black, W.C., Babin, B. J., Anderson, R. E., Tatham, R. L. (2005). Análise multivariada de dados. (5th ed.). Porto Alegre: Bookman.

Karasek, R., et al. (2000). Psychosocial factors: review of the empirical data among men. Occupational Medicine, 15(1).

Jodas, D. A., Haddad, M. C. L. (2009). Síndrome de Burnout em trabalhadores de enfermagem de um pronto socorro de hospital universitário. Acta Paulista de Enfermagem, 22(2), 192-197.

Lazarus, R. S., Folkman, S. (1984). Coping and adaptation. The handbook of behavioral medicine, pp.282-325.

Levi, L. (2003). Sociedade, Stress e Doença - Investimentos para a saúde e desenvolvimento: causas, mecanismos, consequências, prevenção e promoção. In III Congresso de Stress da ISMA - BR (International Stress Management Association) e V Fórum Internacional de Qualidade de Vida no Trabalho. Porto Alegre.

Levi, L. (2005). Sociedade, stress e doença - investimentos para a saúde e desenvolvimento: causas, mecanismos, consequências, prevenção e promoção. In V Congresso de Stress da ISMA - BR (International Stress Management Association) e VII Fórum Internacional de Qualidade de Vida no Trabalho. Porto Alegre.

Levi, L. (2010). O guia da comissão europeia sobre stress relacionado ao trabalho e iniciativas relacionadas: das palavras à ação. In Rossi, A. M. Stress e qualidade de vida no trabalho: perspectivas atuais da saúde ocupacional. São Paulo: Atlas.

Lipp, M. E. N. (2005). Stress e o turbilhão da raiva. São Paulo: Caso do Psicólogo.

Limongi-França, A. C., Rodrigues, A. L. (2005). Stress e trabalho: uma abordagem psicossomática. (4th ed.). São Paulo: Atlas. 
Malhotra, N. (2001). Pesquisa de marketing. (3th ed.). Porto Alegre: Bookman.

Marques, L. A., Borges, R., Reis, I. C. (2016). Mudança organizacional e satisfação no trabalho: um estudo com servidores públicos do estado de Minas Gerais. Revista de Administração Pública, 50(1), 41-58.

Melo, M. C. O. L., Cassini, M. R. O. L., Lopes, A. L. M. (2011). Do estresse e mal-estar gerencial ao surgimento da síndrome de Estocolmo gerencial. Revista Psicologia: Organização e Trabalho, 11(2), 84-99.

Newstrom, J., Santos, I.P.F. (2008). Comportamento organizacional: o comportamento humano no trabalho. São Paulo: McGraw Hill.

Oliveira, E. B., Souza, N. V. M. (2012). Estresse e inovação tecnológica em Unidade de Terapia Intensiva de Cardiologia: Tecnologia Dura. Revista Enfermagem UERJ, 20(4), 457- 462.

Paschoal, T., Tamayo, A. (2004). Validation of the work stress scale. Estudos de Psicologia, 9(1), 45-52.

Pestana, M. H., Gageiro, J. N. (2000). Análise de dados para ciências sociais: a complementariedade do SPSS. (2th ed.). Lisboa: Edições Silabo.

Santos, C. C., Zille, L. P. (2014). Estresse ocupacional: estudo com técnicos de enfermagem em um hospital público federal de Minas Gerais. In XXXVIII Encontro da ANPAD. Rio de Janeiro, 13.

Santos, C. C. (2015). ESTRESSE OCUPACIONAL: estudo com profissionais de enfermagem de um hospital universitário no estado de Minas Gerais. Dissertação de Mestrado não-publicada, Centro Universitário Unihorizontes, Belo Horizonte, Brasil.

Savoia, M. G. (1999). Escalas de eventos vitais e de estratégias de enfrentamento (coping). Revista de Psiquiatria Clínica, 26(2), 57-67.

Selye, H. (1936). A syndrome produced by diverse nocuous agents. Nature, 138(3479), 32-32.

Selye, H. (1959). Stress: a tensão da vida. São Paulo: Ibrasa.

Schermerhorn, J. R., Hunt, J. G., Osborn, R. N. (2009). Fundamentos de comportamento organizacional. São Paulo: Bookman.

Sousa, V. F. S., Araujo, T. C. C. F. (2015). Occupational Stress and Resilience Among Health Professionals. Psicologia: Ciência e Profissão, 35(3), 900-915.

Yin, R. K. Estudo de Caso: planejamento e métodos. (3th ed.). Porto Alegre: Bookman.

Zanelli, L. C. (2010). Estresse nas organizações de trabalho: compreensão e intervenção baseadas em evidencias. Porto Alegre: Artmed.

Zille, L. P. (2005). Novas perspectivas para a abordagem do estresse ocupacional em gerentes: estudo em organizações brasileiras de setores diversos. Tese de Doutorado não-publicada, Centro de Pós-graduação e Pesquisas em Administração, Universidade Federal de Minas Gerais, Belo Horizonte, Brasil.

Zille, L. P., Zille, G. P. (2010). O estresse no trabalho: uma análise teórica de seus conceitos e suas inter-relações. Revista Gestão e Sociedade, 4(7) 414-434.

Zille, L. P., Marques, A. L., Melo, M. C. O. L., Braga, C. D., Zille, G. P. (2011). Tensões excessivas no trabalho e o estresse ocupacional: estudo com gestores que atuam em empresas privadas de setores diversos. In ENCONTRO DE GESTAO DE PESSOAS E RELAÇOES DE TRABALHO, João Pessoa.

* Agradecimento à FAPEMIG pelo apoio no desenvolvimento do estudo. 